\title{
Advancements in therapeutically targeting orphan GPCRs
}

\author{
Jennifer A. Stockert and Lakshmi A. Devi* \\ Department of Pharmacology and Systems Therapeutics, Icahn School of Medicine at Mount Sinai, New York, NY, USA
}

G-protein coupled receptors (GPCRs) are popular biological targets for drug discovery and development. To date there are more than 140 orphan GPCRs, i.e., receptors whose endogenous ligands are unknown. Traditionally orphan GPCRs have been difficult to study and the development of therapeutic compounds targeting these receptors has been extremely slow although these GPCRs are considered important

OPEN ACCESS

Edited by:

Terry Kenakin,

University of North Carolina,

Chapel Hill, USA

Reviewed by:

Suyash Prasad,

Audentes Therapeutics, USA

Gerfried Karl Hans Nell,

NPC Nell Pharma Connect Ltd.,

Austria

*Correspondence:

Lakshmi A. Devi,

Department of Pharmacology and Systems Therapeutics, Icahn School of Medicine at Mount Sinai,

19-84 Annenberg Building, One

Gustave L. Levy Place, New York,

NY 10029, USA

lakshmi.devi@mssm.edu

Specialty section:

This article was submitted to

Pharmaceutical Medicine

and Outcomes Research,

a section of the journal

Frontiers in Pharmacology

Received: 13 February 2015

Paper pending published:

29 March 2015

Accepted: 21 April 2015

Published: 08 May 2015

Citation:

Stockert JA and Devi LA (2015) Advancements in therapeutically

targeting orphan GPCRs.

Front. Pharmacol. 6:100.

doi: 10.3389/fphar.2015.00100 targets based on their distribution and behavioral phenotype as revealed by animals lacking the receptor. Recent advances in several methods used to study orphan receptors, including protein crystallography and homology modeling are likely to be useful in the identification of therapeutics targeting these receptors. In the past 13 years, over a dozen different Class A GPCRs have been crystallized; this trend is exciting, since homology modeling of GPCRs has previously been limited by the availability of solved structures. As the number of solved GPCR structures continues to grow so does the number of templates that can be used to generate increasingly accurate models of phylogenetically related orphan GPCRs. The availability of solved structures along with the advances in using multiple templates to build models (in combination with molecular dynamics simulations that reveal structural information not provided by crystallographic data and methods for modeling hard-to-predict flexible loop regions) have improved the quality of GPCR homology models. This, in turn, has improved the success rates of virtual ligand screens that use homology models to identify potential receptor binding compounds. Experimental testing of the predicted hits and validation using traditional GPCR pharmacological approaches can be used to drive ligand-based efforts to probe orphan receptor biology as well as to define the chemotypes and chemical scaffolds important for binding. As a result of these advances, orphan GPCRs are emerging from relative obscurity as a new class of drug targets.

Keywords: Homology modeling, virtual screening, MD simulations, protein crystallography

Abbreviations: BLAST, basic local alignment search tool; BRIL, apocytochrome b562RIL; cAMP, cyclic AMP; CCR2, C-C chemokine receptor type 2; CysLTR1, cysteinyl leukotriene receptor 1; DAG, diacylglycerol; DARPP-32, dopamine- and cAMP-regulated phosphoprotein of $32 \mathrm{kDa}$; DRD3, dopamine $\mathrm{D}_{3}$ receptor; ECL, extracellular loop; FFAR1, free fatty acid receptor 1; FPR1R, formylpeptide receptor; GABA, gamma-aminobutyric acid; GHS-R, growth hormone secretagogue receptor; GPCR, G-protein coupled receptor; GRAFS, glutamate, rhodopsin, adhesion, frizzled/taste2, secretin; H, helix; HH4R, human histamine H4 receptor; ICL, intracellular loop; Ins(1,4,5)P3, inositol 1,4,5-trisphosphate; KD/KO, knockdown/knockout; MCHR1, melanin-concentrating hormone receptor 1; MDs, molecular dynamic simulations; NK1, neurokinin 1 receptor; N/OFQ, nociception/orphanin FQ receptor; ORL-1, opioid receptor-like 1; $\mathrm{OX}_{1} \mathrm{R}$, orexin receptor type 1; $\mathrm{OX}_{2} \mathrm{R}$, orexin receptor type 2; P2RY5, purinergic receptor 5; PLC $\beta$, phospholipase C $\beta$; PDB, protein data bank; PKA, protein kinase A; PKC, protein kinase C; RMSD, root-mean square deviation; T4L, T4-lysozyme; TM, transmembrane; TRHR, thyrotropin-releasing hormone receptor; VLS, virtual ligand screening. 


\section{Introduction}

G-protein coupled receptors are by far the largest group of TM signaling receptors in the human genome. Phylogenetic analysis has estimated that over 800 genes encode for GPCRs (Fredriksson et al., 2003), which amounts to over $1 \%$ of the entire human genome (Jassal et al., 2010). GPCRs are ubiquitously expressed throughout the body and modulate a wide array of essential physiological processes, including those governing the senses of vision, taste, and olfaction, as well as mediating cellular responses to hormones and neurotransmitters (Rosenbaum et al., 2009). GPCR signal transduction is accomplished by the binding of a receptor agonist to an exposed extracellular or intramembrane site on the receptor, which causes a conformational change in the receptor protein. This leads to the uncoupling of the heterotrimeric G-proteins associated with the receptor which, in turn, unleashes a cascade of intracellular downstream effector molecules. The assortment of known GPCR ligands is as expansive and diverse as the biological functions that they regulate, and includes photons, ions, odorants, amino acids, peptides, nucleotides, lipids, and small organic molecules (Kroeze et al., 2003; Venkatakrishnan et al., 2013). Because of the range of biological processes, and therefore pathologies, that they modulate, GPCRs have been popular and successful biological targets in the history of drug development.

Despite the diversity of this superfamily of proteins, all GPCRs have the same basic structure and signaling mechanisms. Thus the structure of a GPCR can be broken down into three basic parts: (i) the extracellular-facing region comprising the $\mathrm{N}$-terminus and three ECL (ECL1-ECL3), (ii) the TM region, a defining motif of GPCRs, that consists of seven $\alpha$-helices (labeled TM1-TM7) bundled together and which span the width of the membrane at varying lengths and angles, and (iii) the intracellular region that contains three ICL (ICL1-ICL3), an additional helix (H8) and C-tail. While the extracellular domain mediates the access of ligand(s) to the receptor (Venkatakrishnan et al., 2013), the TM region undergoes a conformational change upon the binding of a ligand that is transmitted to the intracellular region of the GPCR (Venkatakrishnan et al., 2013). The latter region is responsible for interacting with downstream effectors such as G-proteins (i.e., $\mathrm{G} \alpha$ and $\mathrm{G} \beta \gamma$ subunits) and arrestins (Katritch et al., 2012). In canonical GPCR signaling, agonist binding to the receptor leads to the activation of G-proteins and these, in turn, activate effector molecules such as adenylyl cyclase and PLC $\beta$ resulting in the production of second messengers such as cAMP, DAG and Ins $(1,4,5) \mathrm{P} 3$, that continue the signaling cascade by activating further downstream proteins including PKA and PKC (Ritter and Hall, 2009), to name a few.

Given the size and scope of the GPCR superfamily, several classification schemes have been proposed. While some of these attempted to group GPCRs based on either the dynamics of ligand binding, physiological, or structural characteristics, the best-known classification scheme categorizes GPCRs based on their sequence and structural similarity (Fredriksson et al., 2003). Thus, phylogenetic analyses of non-olfactory human GPCRs have resulted in their grouping into five distinct classes abbreviated as GRAFS: glutamate (i.e., Family $\mathrm{C}$ receptors) rhodopsin (i.e., Family A receptors), adhesion, Frizzled/Taste2, and secretin (i.e., Family B receptors) receptors (Fredriksson et al., 2003; Rosenbaum et al., 2009; Table 1). Class A rhodopsin-like receptors form the largest, most diverse, and best studied, and contains over 700 receptors; $\sim 240$ members of this family are non-olfactory and include prostaglandin, amine (e.g., serotonin, dopamine, and histamine), and melatonin receptors (Fredriksson et al., 2003). The secretin and glutamate families both contain $\sim 15$ members and include the glucagon and GABA receptors, respectively. The adhesion family, so-called because they contain one or more domains with adhesion-like motifs at the $\mathrm{N}$-terminus, comprises $\sim 24$ members, while the Frizzled/Taste2 family contains $\sim 24$ receptors that mediate cellular events in metazoan development and the sense of taste (Fredriksson et al., 2003).

At present there are over 140 non-olfactory GPCRs for which endogenous ligands have not been identified or whose biological functions remain unknown (Tang et al., 2012). Conventionally it has been considered important to identify the endogenous ligand(s) activating a GPCR in order to study the receptor's signaling/regulatory mechanisms and physiological function. Without this basic information, developing therapeutics targeting these receptors is considered to be nearly impossible (Yoshida et al., 2012). In the case of orphan GPCRs, the lack of knowledge about the endogenous ligand has focused research on the identification of cognate GPCR-ligand pairs rather than lead-compound identification and drug discovery.

Orphan receptors that have been matched to their endogenous ligand are termed deorphanized receptors. The earliest attempts to deorphanize GPCRs began in the latter half of the 1980s, and this coincided with advances in molecular biological techniques including low-stringency hybridization and degenerate polymerase chain reaction, which allowed for the successful cloning of orphan GPCRs (Civelli et al., 2013). Moreover, modern cloning technology allowed researchers to express orphan GPCRs in cell lines so that they could be tested in signal transduction assays with potential exogenous or endogenous ligands (Civelli et al., 2013). This strategy known as "reverse pharmacology" was used in the 1990s to deorphanize many ligand-receptor systems, including nociception for ORL-1, orexin- $\mathrm{A}$ for $\mathrm{OX}_{1} \mathrm{R}$, orexin-B for $\mathrm{OX}_{2} \mathrm{R}$, and ghrelin for GHS-R (Yoshida et al., 2012). The

TABLE 1 | Solved structures of the GPCR families.

\begin{tabular}{lll}
\hline GPCR family & $\begin{array}{l}\text { Identified member } \\
\text { receptors }\end{array}$ & $\begin{array}{l}\text { Solved receptor } \\
\text { structures }\end{array}$ \\
\hline Rhodopsin & 701 & 21 \\
Adhesion & 24 & 0 \\
Frizzled/Tas2 & 24 & 1 \\
Glutamate & 15 & 1 \\
Secretin & 15 & 2
\end{tabular}

The largest GPCR family, Rhodopsin, has the largest number of solved structures. Since the first structure of a GPCR, bovine rhodopsin, was published in 2000, 24 other GPCR structures have been solved, indicative of the acceleration in solving GPCR structures. 
endogenous ligands were then used to further examine the pharmacological and physiological properties of the receptor in vitro or in vivo (Yoshida et al., 2012).

\section{Orphan GPCRs as Therapeutic Targets}

Studies examining the distribution and localization as well as studies probing the behavioral phenotype of animals lacking the orphan GPCRs have been central to establishing the particular receptor as an attractive therapeutic target. Studies examining receptor expression by in situ hybridization and studies investigating the phenotypic characterization of targeted $\mathrm{KD} / \mathrm{KO}$ of orphan GPCRs have proven extremely useful in elucidating their biological functions, and in suggesting their role as potential drug targets. For example, a study with the orphan receptor GPR88 used molecular and behavioral tests to propose a role for this receptor in schizophrenia (Logue et al., 2009). GPR88 mRNA was found to be highly expressed in the striatum of WT mice brains and absent in mice lacking GPR88 (GPR88 KO) using in situ hybridization (Logue et al., 2009). GPR88 KO mice had higher levels of phosphorylated DARPP-32 and increased sensitivity to dopamine, suggesting that GPR88 may play an important role in striatal function and dopamine response, making this orphan receptor a potential drug target for the treatment of psychiatric disorders involving the striatum like schizophrenia. Another study with the orphan GPR161 (also known as RE2) proposed a role for this receptor in the proper formation of the tubes of the heart (Leung et al., 2008). In this case in situ hybridization with developing embryos revealed GPR161 mRNA expression in the precardiac mesoderm, and knock-down of GPR161 resulted not only in pericardial edema, improper positioning of the ventricle and atrium, malformation of cardiac loops, and left-right (LR) patterning, but also elevated $\mathrm{Ca}^{2+}$ levels in Kupffer's vesicle (an organ in zebrafish that regulates L-R in the heart; Leung et al., 2008). While further studies are needed to characterize this receptor system (i.e., identify downstream signaling pathways), the results of this study indicate that GPR161 may be a therapeutic target for the treatment of congenital heart defects. However the lack of information regarding their endogenous ligands or signaling pathways activated hindered the efforts to identify therapeutics targeting these orphan GPCRs. Advances in homology modeling based on protein crystal structure and in silico screening techniques have begun to be applied toward identification of exogenous ligands (to be developed as therapeutics) targeting orphan GPCRs.

\section{Protein Crystallography}

The first reported structure of a GPCR, bovine rhodopsin, was published in 2000 (Palczewski et al., 2000) and was considered a landmark achievement for crystallographers and GPCR biologists. It confirmed that the TM region of GPCRs contains seven $\alpha$-helices and can serve as a template for other GPCRs, allowing researchers to deduce the location of secondary structural components and highly conserved sequences on related receptors (Palczewski et al., 2000). Successive crystallizations of rhodopsin in various active and inactive conformations with its ligand also provided insights into the mechanisms surrounding GPCR activation (Weis and Kobilka, 2008). Because of the inherent difficulties in crystallizing proteins, it took 7 years for the next GPCR to be crystallized, which was the human $\beta_{2}$-adrenergic receptor (Cherezov et al., 2007). Since 2007, there have been several breakthroughs in crystallization techniques which have led to a dramatic increase in the number of GPCR structures available. One such technique involves engineering GPCRs to contain small soluble proteins that crystallize under known conditions, in the hope that this will increase the number of lattice contacts necessary for crystal formation between protein molecules (Rosenbaum et al., 2007). This innovative idea was first employed in the crystallization of the $\beta_{2}$-adrenergic receptor, where the C-terminus and ICL3 of the receptor were replaced with a protein, T4L (Cherezov et al., 2007). A similar strategy was used to crystallize the N/OFQ receptor in 2012 (Thompson et al., 2012), where again the GPCR was modified by fusing a stable protein (in this case, BRIL; Chu et al., 2002) to a truncated $\mathrm{N}$-terminus to facilitate crystal formation (Cherezov et al., 2007). Crystallization has also been accomplished with the help of antibodies, as seen with an additional structure of the $\beta_{2}$-adrenergic receptor (Rasmussen et al., 2007). Other techniques used to facilitate GPCR crystallization include the use of new detergents and engineering mutations into the receptors (Venkatakrishnan et al., 2013). As a result of these advances, as of January 2015 there are 25 evolutionarily diverse crystal structures of GPCRs available (Classes A, B, C, and Frizzled), spread across various classes (see Table 1; Tautermann, 2014). These atomic-level, high-resolution insights into GPCR structure are invaluable in the generation of homology models that will aid in the development of rational, structure-based design of therapeutics targeting GPCRs.

\section{Protein Structures and Homology Modeling}

The modeling process involves several steps beginning with the selection of the protein to be modeled and the template protein, to aligning their primary amino acid sequences and making appropriate corrections, to initial model generation and later refinements, and ending with the validation of the model (Krieger et al., 2003). The most important part of this process is the selection of the appropriate homolog to serve as the template.

Once the protein to be modeled has been chosen (i.e., the target protein) the search for a related protein with known structure (i.e., the template) is accomplished by using sequence-based methods. There are several repositories and structure databases available online, and among them the $\mathrm{PDB}^{1}$ is the most popular (Berman et al., 2000). The published structures for proteins are stored at PDB and are easily accessible. Search algorithms such as BLAST (Altschul et al., 1990), compare the entered target's DNA or amino acid sequence to the sequences of other

${ }^{1}$ www.rcsb.org 
proteins, identify regions of similarity between them, return a list of proteins ranked in order of their similarity to the query sequence, and also display the alignment (Altschul et al., 1990). Sequences that are more than $40 \%$ identical and are found using tools such as BLAST are usually correctly aligned (Saxena et al., 2013), however, errors in the initial alignment are common if the sequences share less than $30 \%$ identity (Rost, 1999). It is important to note that BLAST will not work on difficult targets (proteins with only distantly related homologs), and more sensitive method(s) are needed (such as those that compare multiple sequence alignments) in these instances. While it is possible to optimize the initial alignment using specialized alignment software (Nayeem et al., 2006), currently there are no modeling programs available that can construct an accurate model from an incorrect alignment (Sanchez and Sali, 1997) and therefore every attempt should be made to correctly align the target and template sequences.

To model proteins such as GPCRs, it is crucial that there be an adequate supply of highly resolved, diverse GPCR crystal structures that can serve as templates. The advances in GPCR protein crystallization, mentioned previously, have generated a cache of templates for modelers to choose from. Information about the list of published structures is available at http://zhanglab.ccmb. med.umich.edu/GPCR-EXP/ and a visualization of the phylogenetic tree of GPCRs shows the diversity of available structures ${ }^{2}$ (Stevens et al., 2013) and is illustrated in Table $\mathbf{1 .}$

\section{Advances in Homology Modeling of GPCRs}

With the increase in the number of templates available for GPCRs there has been renewed interest in improving the methodologies and techniques that are used to create homology models. These include multi-template modeling approaches, MDs, and the treatment of long loop regions in the receptors (Yarnitzky et al., 2010). These are described below.

As its name suggests, multi-template modeling involves the use of more than one template to create a model of the target. The effect of using multiple templates to generate models of the $\beta_{2}$-adrenergic receptor was evaluated in one paper by comparing the RMSD of ligand poses in multiple and singular template models of the receptor compared to its crystal structure (Mobarec et al., 2009). The multi-template model performed slightly better than the single-template models, since it had a slightly higher percentage of docking poses of the ligand with an RMSD less than $2 \AA$ compared to the crystal structure (Mobarec et al., 2009). However, it should be noted that this improvement was only observed when all the templates used shared low sequence similarity to the target; if the templates had a relatively high sequence similarity to the target, there was no significant difference in the performance of multi-template and single-template models (Mobarec et al., 2009). Another example of successful multi-template modeling is in the enrichment of VLS of homology models of the

${ }^{2}$ http://gpcr.scripps.edu/
NK1 receptor (Kneissl et al., 2009). In this study, models of NK1 were built using either bovine rhodopsin or human $\beta_{2}$ adrenergic receptor as a template, or using a combination of bovine rhodopsin and $\beta_{2}$-adrenergic receptor as template (Kneissl et al., 2009). NK1 models built based on only one template did not conform to experimental mutagenesis data and needed further refinements; however, the multiple-template model achieved enriched VLS scores in agreement with a consensus model without any additional refinements (Kneissl et al., 2009).

One of the more problematic aspects of modeling GPCRs is in the treatment of variable loop regions. The ECLs and ICLs between GPCRs are much less conserved and often differ between target and template, which makes them extremely difficult to model using template-based approaches (Goldfeld et al., 2011). This is perhaps the greatest disadvantage of using template-based homology models because these loop regions are extremely important in ligand binding and receptor activation (Goldfeld et al., 2011). The ECLs of GPCRs have been shown to facilitate the binding of ligands of several different chemotypes including small drug-like molecules (de Graaf et al., 2008), large proteins (de Graaf et al., 2008), and various low molecular weight biological compounds (Goldfeld et al., 2011) and can also regulate receptor activation (Klco et al., 2005). ICLs group together to form functional domains that interact with the G-proteins coupled to the receptor (Wong, 2003). Because these regions are so integral to proper GPCR function much effort has gone into improving the modeling of these unpredictable peptide sequences, and what to do if loop modeling proves too complex. For this the modeling field has moved toward more template-free approaches that use energy functions to guide loop folding in a defined conformational space (Li, 2013). During this process a large number of models are generated and the best models are chosen for further refinement based on their minimized energy in terms of geometric constraints (i.e., minimized steric constraints, etc.; Li, 2013). The success of this method relies on the ability of algorithms to effectively sample the best conformations and evaluate model energy (Tang et al., 2014). Since 2000 several programs have been developed for loop modeling and each uses different energy functions and sampling criteria (Li, 2013). Some popular programs among many include MODELLER (uses statistical potentials to integrate restraints or pseudoenergy; Fiser et al., 2000), LOOPY (uses colony energy; Xiang et al., 2002), RAPPER (uses AMBER force field combined with the Generalized Born solvation model; de Bakker et al., 2003), Rosetta (uses Rosetta; Rohl et al., 2004), and Looper (uses CHARMM force field with additional parameters; Spassov et al., 2008). In general these programs are very effective at modeling loops as long as eight residues ( $\mathrm{Li}, 2013$ ), and newer methods can accurately predict loop structures up to 13 residues long (Mandell et al., 2009; Li et al., 2011), but modeling longer loops still remains a significant challenge $(\mathrm{Li}$, 2013).

In recent years homology models of orphan GPCRs have been generated. For example, a model for GPR18, a Class A receptor that might play a role in apoptosis, using the 
$\mathrm{N} / \mathrm{OFQ}$ receptor as a template has been generated (Kothandan and Cho, 2013). Also two homology models were generated to examine the effects of mutations on the structure of the P2RY5 receptor, an orphan GPCR identified by genetic linkage analysis that is implicated in autosomal recessive wooly hair (Shimomura et al., 2008). In addition, a homology model of GPR55 was built using the crystal structure of the adenosine A (2A) receptor and used to dock known ligands (Elbegdorj et al., 2013).

Molecular dynamic simulations are time-dependent computer-based simulations that track the movement of atoms and molecules in a defined system (Lindahl, 2008). When MDs are applied to large proteins such as GPCRs they can reveal important structural information, and in general serve as a means to assess how theoretical models (such as homology models) compare to experimental data (Lindahl, 2008). In the case of GPCRs, MDs are recorded on a timescale ranging from nanoseconds to microseconds and can be used to study not only how a ligand reaches the binding pocket of a GPCR but also the mechanism of receptor activation, oligomerization, and allosteric modulation (Bruno and Costantino, 2012). In homology modeling MDs are used for further refinement to optimize protein folding (Yarnitzky et al., 2010) thereby improving the performance of the model for ligand docking simulations (Yarnitzky et al., 2010). In one study bovine rhodopsin was used as a template to create a model of the HH4R, and MDs were run on the receptor in a membrane-bound environment with and without its agonist histamine and a selective antagonist (Jójárt et al., 2008). The simulations modeled receptor conformational changes and histamine-binding site interactions that agreed with previous experimental data (Jójárt et al., 2008). In addition, the modeled movements of several TMs were also in agreement with experimental data. Taken together these results indicated that the MDs of the HH4R can serve as a structural model for identification of HH4R ligands (Jójárt et al., 2008).

Molecular dynamic simulations can also be employed in an innovative way to address some problems associated with ligand binding to homology models. For example, models built using the bovine rhodopsin template are sometimes unable to bind to known ligands because the binding site is too small. In this case pressure-guided molecular dynamics can be used to correct this problem (Kimura et al., 2008). During these MDs, pressure is applied to the binding site of the receptor to expand it much in the same way that a balloon expands when filled with air and for this reason this is sometimes called the "balloon expansion method" (Kimura et al., 2008). To test the legitimacy of this method, a structure of bovine rhodopsin was created without its ligand retinal, rendering it inactive and closed. Upon application of the "balloon pressure" retinal was able to bind almost exactly as it is known to (Kimura et al., 2008). When tested on an unrefined homology model of the chemokine receptor CCR2 that was originally unable to bind three known antagonists, the applied pressure allowed for the ligands to bind in a manner that agreed with mutagenesis data (Kimura et al., 2008).

\section{In Silico Screening Using Homology Models of GPCRs for Drug Discovery}

One of the most practical and popular applications of homology models is their use in in silico virtual screens for drug discovery. An example of successful use of homology modeling to identify small molecule ligands is that of DRD3. In this case, a homology model of the DRD3, built using the $\beta_{2}$-adrenergic receptor as a template, was used to dock over 3 million compounds (Carlsson et al., 2011). When the 26 top-scoring molecules were tested for binding affinity, six of these compounds exhibited affinities in the micromolar range (Carlsson et al., 2011). Soon after this initial screen, the crystal structure for the DRD3 receptor was released and the same docking experiment was performed; this yielded five molecules with affinities between nanomolar and micromolar concentrations (Carlsson et al., 2011). The hit rates between model and crystal structure were also similar, at 23 and $20 \%$, respectively. What is remarkable about this study is that there was no difference in the screening capability between the homology model and crystal structure; the model was able to identify binding ligands at the same rate as the crystal structure (Carlsson et al., 2011). While this study demonstrates the effectiveness of homology models in virtual screens, this was not the first time that homology models were successfully used to predict novel ligands for GPCRs. Homology models of the HH4R (Kiss et al., 2008), FFAR1 (Tikhonova et al., 2008), and MCHR1 (Cavasotto et al., 2008), among other examples (Edwards et al., 2005; Engel et al., 2008) were able to identify novel binding compounds that exhibited either agonistic or antagonistic properties (Table 2). A recent review (Kooistra et al., 2013) lists over 15 examples of successful structure-based virtual screens of GPCR homology models (Table 2 summarizes six of these virtual screens). The success with the identification of these ligands clearly demonstrates the usefulness of homology models in ligand discovery. Virtual docking approaches similar to those used in the case of models of known GPCRs are likely to be useful in the identification of ligands targeting orphan GPCRs.

As described above, while the GPCR homology models have been used to study structural features of orphan receptors, homology modeling has not yet been widely applied in the context of in silico drug screens. Perhaps the best example of a successful in silico compound screen for an orphan receptor is in the case of GPR17. A homology model of the receptor was created using the multi-template approach by combining the crystal structures of four GPCRs, and was then used to screen an in-house chemical library (the Asinex Platinum Collection) containing 130,000 lead-like compounds (Eberini et al., 2011). The binding site of the receptor was identified using the MOE Site Finder module, which evaluates possible binding sites according to their geometry and location; it is a ligand-free approach and does not use known binding compounds to detect the binding site (Eberini et al., 2011). The top-5 scoring compounds (compounds binding with the lowest energy conformations) were purchased and receptor activation was measured in dose-response $\left[{ }^{35} \mathrm{~S}\right]$ GTP $\gamma S$ binding assays (Eberini et al., 2011). Four of these compounds displayed full agonist activity and one compound 
TABLE 2 | Novel ligands predicted by virtual screening against GPCR homology models.

\begin{tabular}{|c|c|c|c|}
\hline Receptor & Ligand with highest affinity & Agonist or antagonist & Reference \\
\hline DRD3 & & Antagonist $K_{\mathrm{i}}=0.3 \mu \mathrm{M}$ & Carlsson et al. (2011) \\
\hline $\mathrm{HH} 4 \mathrm{R}$ & & Antagonist $K_{\mathrm{i}}=85 \mathrm{nM}$ & Kiss et al. (2008) \\
\hline FFAR1 & & Agonist $E C_{50}=3.6 \mu \mathrm{M}$ & Tikhonova et al. (2008) \\
\hline FPR1R & & Partial agonist $K_{\mathrm{i}}=\mid \mu \mathrm{M}$ & Edwards et al. (2005) \\
\hline
\end{tabular}

was shown to act as a partial agonist, as compared to a reference ligand; all compounds exhibited nanomolar or sub-nanomolar potencies (Eberini et al., 2011). This virtual screen was also able to identify efficacious molecules with novel chemical scaffolds; these compounds are the first examples of GPR17 ligands that are not derived from the structures of previously known GPR17 activators (i.e., CysLTR1 or $\mathrm{P}_{2}$ YR ligands; Eberini et al., 2011). While GPR17 is not a strictly orphan receptor, almost no information regarding known ligands was needed to execute this study (except for activity characterization/comparison in the in vitro experiments) and it therefore serves as a good example of novel and efficacious lead-compound discovery at a virtually unknown receptor system.

\section{Conclusion}

GPCRs are the most targeted sites for drug discovery and this trend is not predicted to end soon. Moreover, breakthroughs in protein crystallization strategies and computer technology have opened the doors for in silico methods of studying these receptors. This includes advances in GPCR crystallization that have led to the release of many new crystal structures, igniting renewed interest in the structure-based study of GPCRs. Thus GPCR homology modelers in need of templates now have dozens of functionally and evolutionary diverse options to choose from. This taken together with the recent advances in the computational aspects of modeling make GPCR homology models more accurate. As a result, virtual ligand screens using these models have successfully identified both known ligands and novel compounds. The performance of GPCR homology models in virtual screens compared to crystal structures is impressive, and clearly validates their continued use in ligand discovery. This bodes well for the homology modeling of orphan GPCRs and the use of modern in silico screening methods to identify putative ligands, both agonists and antagonists that could be used to elucidate the physiological role of the receptor. 
The combination of computational and biological methods provides a unique approach to studying orphan GPCRs that offers several advantages over strictly biological approaches. Thus if computational methods are applied to orphan receptors, they provide an unbiased approach at ligand discovery which enhances the chances for successful discovery of novel compounds, and novel chemical scaffolds. Moreover, while biological methods are limited by the amount of resources (cells, compounds, etc.) available for receptor ligand screening, in silico screening allows for the testing of $1000 \mathrm{~s}$ of compounds without costly in vivo or in vitro screening. As it

\section{References}

Altschul, S. F., Gish, W., Miller, W., Meyers, E. W., and Lipman, D. J. (1990). Basic local alignment search tool. J. Mol. Biol. 215, 403-410. doi: 10.1016/S00222836(05)80360-2

Berman, H. M., Westbrook, J., Feng, Z., Gilliland, G., Bhat, T. N., Weissig, H., et al. (2000). The Protein data bank. Nucleic Acids Res. 28, 235-242. doi: 10.1093/nar/28.1.235

Bruno, A., and Costantino, G. (2012). Molecular dynamics simulations of $\mathrm{G}$ protein-coupled receptors. Mol. Inform. 31, 222-230. doi: 10.1002/minf.201100138

Carlsson, J., Coleman, R. G., Setola, V., Irwin, J. J., Fan, H., Schlessinger, A., et al. (2011). Ligand discovery from a dopamine D3 receptor homology model and crystal structure. Nat. Chem. Biol. 7, 769-778. doi: 10.1038/nchembio.662

Cavasotto, C. N., Orry, A. J., Murgolo, N. J., Czarniecki, M. F., Kocsi, S. A., Hawes, B. E., et al. (2008). Discovery of novel chemotypes to a G-protein-coupled receptor through ligand-steered homology modeling and structure-based virtual screening. J. Med. Chem.51, 581-588. doi: 10.1021/jm070759m

Cherezov, V., Rosenbaum, D. M., Hanson, M. A., Rasmussen, S. G. F., Thian, F. S., Kobilka, T. S., et al. (2007). High-resolution crystal structure of an engineered human $\beta 2$-adrenergic G protein-coupled receptor. Science 318, 1258-1265. doi: 10.1126/science.1150577

Chu, R., Takei, J., Knowlton, J. R., Andrykovitch, M., Pei, W., Kajava, A. V., et al. (2002). Redesign of a four-helix bundle protein by phage display coupled with proteolysis and structural characterization by NMR and X-ray crystallography. J. Mol. Biol. 323, 253-262. doi: 10.1016/S0022-2836(02)00884-7

Civelli, O., Reinscheid, R. K., Zhang, Y., Wang, Z., Fredriksson, R., and Schiöth, H. B. (2013). G protein-coupled receptor deorphanizations. Annu. Rev. Pharmacol. Toxicol. 53, 127-146. doi: 10.1146/annurev-pharmtox-010611134548

de Bakker, P. I., DePristo, M. A., Burke, D. F., and Blundell, T. L. (2003). Ab initio construction of polypeptide fragments: accuracy of loop decoy discrimination by an all-atom statistical potential and the AMBER force field with the Generalized Born solvation model. Proteins 51, 21-40. doi: 10.1002/prot.10235

de Graaf, C., Foata, N., Engkvist, O., and Rognan, D. (2008). Molecular modeling of the second extracellular loop of G-protein coupled receptors and its implication on structure-based virtual screening. Proteins 71, 599-620. doi: $10.1002 /$ prot. 21724

Eberini, I., Daniele, S., Parravicini, C., Sensi, C., Trincavelli, M. L., Martini, C., et al. (2011). In silico identification of new ligands for GPR17: a promising therapeutic target for neurodegenerative diseases. J. Comput. Aided Mol. Des. 25, 743-752. doi: 10.1007/s10822-011-9455-8

Edwards, B. S., Bologa, C., Young, S. M., Balakin, K. V., Prossnitz, E. R., Savchuck, N. P., et al. (2005). Integration of virtual screening with high-throughput flow cytometry to identify novel small molecule formylpeptide receptor antagonists. Mol. Pharmacol. 65, 1301-1310. doi: 10.1124/mol.105.014068

Elbegdorj, O., Westkaemper, R. B., and Zhang, Y. (2013). A homology modeling study toward the understanding of three-dimensional structure and putative pharmacological profile of the G-protein coupled receptor GPR55. J. Mol. Graph. Model. 39, 50-60. doi: 10.1016/j.jmgm.2012. 10.005

Engel, S., Skoumbourdis, A. P., Childress, J., Neumann, S., Deschamps, J. R., Thomas, C. J., et al. (2008). A virtual screen for diverse ligands: discovery stands, ligand discovery for orphan GPCRs via computational methods and validation of lead compounds is ripe for exploration, and stands to be at the cutting edge of orphan GPCR research.

\section{Acknowledgments}

We would like to thank Avner Schlessinger and Ivone Gomes for critically reading the manuscript. This study was supported by DA008863 and DA019521 to LD.

of selective G protein-coupled receptor antagonists. J. Am. Chem. Soc. 130, 5115-5123. doi: 10.1021/ja0776201

Fiser, A., Do, R. K., and Sali, A. (2000). Modeling of loops in protein structures. Protein Sci. 9, 1753-1773. doi: 10.1110/ps.9.9.1753

Fredriksson, R., Lagerström, M. C., Lundin, L. G., and Schiöth, H. B. (2003). The G-protein-coupled receptors in the human genome form five main families. Phylogenetic analysis, paralogon groups, and fingerprints. Mol. Pharmacol. 63, 1256-1272. doi: 10.1124/mol.63.6.1256

Goldfeld, D. A., Zhu, K., Beuming, T., and Friesner, R. A. (2011). Successful prediction of the intra- and extracellular loops of four G-protein-coupled receptors. Proc. Natl. Acad. Sci. U.S.A. 109, 8275-8280. doi: 10.1073/pnas. 1016951108

Jassal, B., Jupe, S., Caudy, M., Birney, E., Stein, L., Hermjakob, H., et al. (2010). The systematic annotation of the three main GPCR families in Reactome. Database (Oxford) 2010:baq018. doi: 10.1093/database/baq018

Jójárt, B., Kiss, R., Viskolcz, B., and Keseru, G. M. (2008). Activation mechanism of the human histamine $\mathrm{H} 4$ receptor-an explicit membrane molecular dynamics simulation study. J. Chem. Inf. Model. 48, 1199-1210. doi: 10.1021/ci700450w

Katritch, V., Cherezov, V., and Stevens, R. C. (2012). Diversity and modularity of G protein-coupled receptor structures. Trends Pharmacol. Sci. 33, 17-27. doi: 10.1016/j.tips.2011.09.003

Kimura, S. R., Tebben, A. J., and Langley, D. R. (2008). Expanding GPCR homology model binding sites via a balloon potential: a molecular dynamics refinement approach. Proteins 71, 1919-1929. doi: 10.1002/prot.21906.

Kiss, R., Kiss, B., Könczöl, A., Szalai, F., Jelinek, I., László, V., et al. (2008). Discovery of novel human histamine H4 receptor ligands by largescale structure-based virtual screening. J. Med. Chem. 51, 3145-3153. doi: $10.1021 /$ jm7014777

Klco, J. M., Wiegand, C. B., Narzinski, K., and Baranski, T. J. (2005). Essential role for the second extracellular loop in C5a receptor activation. Nat. Struct. Mol. Biol. 12, 320-326. doi: 10.1038/nsmb913

Kneissl, B., Leonhardt, B., Hildebrandt, A., and Tautermann, C. S. (2009). Revisiting automated G-protein coupled receptor modeling: the benefit of additional template structures for a neurokinin-1 receptor model. J. Med. Chem. 52, 3166-3173. doi: 10.1021/jm8014487

Kooistra, A. J., Roumen, L., Leurs, R., de Esch, I. J., and de Graaf, C. (2013). From heptahelical bundle to hits from the Haystack: structure-based virtual screening for GPCR ligands. Methods Enzymol. 522, 279-336. doi: 10.1016/B978-0-12407865-9.00015-7

Kothandan, G., and Cho, S. J. (2013). Homology modeling of GPR18 receptor, an orphan G-protein-coupled receptor. J. Chosun Nat. Sci. 6, 16-20. doi: 10.13160/ricns.2013.6.1.016

Krieger, E., Nabuurs, S. B., and Vriend, G. (2003). Homology modeling. Methods Biochem. Anal. 44, 509-523.

Kroeze, W. K., Sheffler, D. J., and Roth, B. L. (2003). G-protein-coupled receptors at a glance. J. Cell Sci. 116, 4867-4869. doi: 10.1242/jcs.00902

Leung, T., Humbert, J. E., Stauffer, A. M., Giger, K. E., Chen, H., Tsai, H. J., et al. (2008). The orphan $G$ protein-coupled receptor 161 is required for left-right patterning. Dev. Biol. 323, 31-40. doi: 10.1016/j.ydbio.2008. 08.001

Li, Y. (2013). Conformational sampling in template-free protein loop structure modeling: an overview. Comput. Struct. Biotechnol. J. 5:e201302003. doi: $10.5936 /$ csbj. 201302003 
Li, Y., Rata, I., and Jakobsson, E. (2011). Sampling multiple scoring functions can improve protein loop structure prediction accuracy. J. Chem. Inf. Model. 51, 1656-1666. doi: 10.1021/ci200143u

Lindahl, E. (2008). Molecular dynamics simulations. Methods Mol. Biol. 1215, 3-26. doi: 10.1007/978-1-4939-1465-4_1

Logue, S. F., Grauer, S. M., Paulsen, J., Graf, R., Taylor, N., Sung, M. A., et al. (2009). The orphan GPCR, GPR88, modulates function of the striatal dopamine system: a possible therapeutic target for psychiatric disorders? Mol. Cell. Neurosci.42, 438-447. doi: 10.1016/j.mcn.2009.09.007

Mandell, D. J., Coutsias, E. A., and Kortemme, T. (2009). Sub-angstrom accuracy in protein loop reconstruction by robotics-inspired conformational sampling. Nat. Methods 6, 551-552. doi: 10.1038/nmeth0809-551

Mobarec, J. C., Sanchez, R., and Filizola, M. (2009). Modern homology modeling of G-protein coupled receptors: which structural template to use? J. Med. Chem. 52, 5207-5216. doi: 10.1021/jm9005252

Nayeem, A., Sitkoff, D., and Krystek, S. Jr. (2006). A comparative study of available software for high-accuracy homology modeling: from sequence alignments to structural models. Protein Sci. 15, 808-824. doi: 10.1110/ps.051892906

Palczewski, K., Kumasaka, T., Hori, T., Behnke, C. A., Motoshima, H., Fox, B. A., et al. (2000). Crystal structure of rhodopsin: a G protein-coupled receptor. Science 289, 739-745. doi: 10.1126/science.289.5480.739

Rasmussen, S. G. F., Choi, H., Rosenbaum, D. M., Kobilka, T. S., Thian, F. S., Edwards, P. C., et al. (2007). Crystal structure of the human $\beta 2$ adrenergic G-protein-coupled receptor. Nature 450, 383-387. doi: 10.1038/nature06325

Ritter, S. L., and Hall, R. A. (2009). Fine-tuning of GPCR activity by receptor-interacting proteins. Nat. Rev. Mol. Cell Biol. 10, 819-830. doi: $10.1038 / \mathrm{nrm} 2803$

Rohl, C. A., Strauss, C. E., Chivian, D., and Baker, D. (2004). Modeling structurally variable regions in homologous proteins with Rosetta. Proteins 55, 656-677. doi: 10.1002/prot.10629

Rosenbaum, D. M., Cherezov, V., Hanson, M. A., Rasmussen, S. G. F., Thian, F. S., Kobilka, T. S., et al. (2007). GPCR engineering yields high-resolution structural insights into $\beta 2$-adrenergic receptor function. Science 318, 1266-1273. doi: 10.1126/science.1150609

Rosenbaum, D. M., Rasmussen, S. G. F., and Kobilka, B. K. (2009). The structure and function of G-protein-coupled receptors. Nature 459, 356-363. doi: 10.1038 /nature08144

Rost, B. (1999). Twilight zone of protein sequence alignments. Protein Eng. 12, 85-94. doi: 10.1093/protein/12.2.85

Sanchez, R., and Sali, A. (1997). Advances in comparative protein-structure modelling. Curr. Opin. Struct. Biol. 7, 206-214. doi: 10.1016/S0959-440X(97)8 0027-9

Saxena, A., Sangwan, R. S., and Mishra, S. (2013). Fundamentals of homology modeling steps and comparison among important bioinformatics tools: an overview. Sci. Int. 1, 237-252. doi: 10.5567/sciintl.2013.237.252

Shimomura, Y., Wajid, M., Ishii, Y., Shapiro, L., Petukhova, L., Gordon, D., et al. (2008). Disruption of P2RY5, an orphan G protein-coupled receptor, underlies autosomal recessive woolly hair. Nat. Genet. 40, 335-339. doi: 10.1038/ng.100

Spassov, V. Z., Flook, P. K., and Yan, L. (2008). LOOPER: a molecular mechanicsbased algorithm for protein loop prediction. Protein Eng. Des. Sel. 21, 91-100. doi: 10.1093/protein/gzm083
Stevens, R. C., Cherezov, V., Katritch, V., Abagyan, R., Kuhn, P., Rosen, H., et al. (2013). The GPCR Network: a large-scale collaboration to determine human GPCR structure and function. Nat. Rev. Drug Discov. 12, 25-34. doi: 10.1038/nrd3859

Tang, K., Zhang, J., and Liang, J. (2014). Fast protein loop sampling and structure prediction using distance-guided sequential chain-growth Monte Carlo method. PLoS Comput. Biol. 10:e1003539. doi: 10.1371/journal.pcbi. 1003539

Tang, X. L., Wang, Y., Li, D. L., Luo, J., and Liu, M. Y. (2012). Orphan $\mathrm{G}$ protein-coupled receptors (GPCRs): biological functions and potential drug targets. Acta Pharmacol. Sin. 33, 363-371. doi: 10.1038/aps. 2011.210

Tautermann, C. S. (2014). GPCR structures in drug design, emerging opportunities with new structures. Bioorg. Med. Chem. Lett. 24, 4073-4079. doi: 10.1016/j.bmcl.2014.07.009

Thompson, A. A., Liu, W., Chun, E., Katritch, V., Wu, H., Vardy, E., et al. (2012). Structure of the nociceptin/orphanin FQ receptor in complex with a peptide mimetic. Nature 485, 395-399. doi: 10.1038/nature11085

Tikhonova, I. G., Sum, C. S., Neumann, S., Engel, S., Raaka, B. M., Costanzi, S., et al. (2008). Discovery of novel agonists and antagonists of the free fatty acid receptor 1 (FFAR1) using virtual screening. J. Med. Chem. 51, 625-633. doi: $10.1021 / \mathrm{jm} 7012425$

Venkatakrishnan, A. J., Deupi, X., Lebon, G., Tate, C. G., Schertler, G. F., and Babu, M. M. (2013). Molecular signatures of G-protein-coupled receptors. Nature 494, 185-194. doi: 10.1038/nature11896

Weis, W. I., and Kobilka, B. K. (2008). Structural insights into G-proteincoupled receptor activation. Curr. Opin. Struct. Biol. 18, 734-740. doi: 10.1016/j.sbi.2008.09.010

Wong, S. K. (2003). G protein selectivity is regulated by multiple intracellular regions of GPCRs. Neurosignals 12, 1-12. doi: 10.1159/ 000068914

Xiang, Z., Soto, C. S., and Honig, B. (2002). Evaluating conformational free energies: the colony energy and its application to the problem of loop prediction. Proc. Natl. Acad. Sci. U.S.A. 99, 7432-7437. doi: 10.1073/pnas.102179699

Yarnitzky, T., Levit, A., and Niv, M. Y. (2010). Homology modeling of G-proteincoupled receptors with X-ray structures on the rise. Curr. Opin. Drug Discov. Devel. 13, 317-325.

Yoshida, M., Miyazato, M., and Kangawa, K. (2012). Orphan GPCRs and methods for identifying their ligands. Methods Enzymol. 514, 33-44. doi: 10.1016/B9780-12-381272-8.00002-7

Conflict of Interest Statement: The authors declare that the research was conducted in the absence of any commercial or financial relationships that could be construed as a potential conflict of interest.

Copyright (C) 2015 Stockert and Devi. This is an open-access article distributed under the terms of the Creative Commons Attribution License (CC BY). The use, distribution or reproduction in other forums is permitted, provided the original author(s) or licensor are credited and that the original publication in this journal is cited, in accordance with accepted academic practice. No use, distribution or reproduction is permitted which does not comply with these terms. 